\title{
Treatment Pathways as Petri Nets in Patient Workflow Management
}

\author{
Svetlana Boudko \\ Norsk Regnesentral \\ Oslo, Norway \\ svetlana.boudko@nr.no
}

\author{
Wolfgang Leister \\ Norsk Regnesentral \\ Oslo, Norway \\ wolfgang.leister@nr.no
}

\begin{abstract}
The healthcare market demands more advanced and flexible solutions to provide better care for patients. Moving medical services closer to patients improves the quality of healthcare but brings, at the same time, new challenges. It requires a well-functioning, decentralized infrastructure and better workflow support for health personnel. Therefore, we need to provide consistency for this type of services with patient data accessed and processed by several healthcare stakeholders.

In the paper, we present how Petri net-based workflow technology can be used to design flexible treatment pathways, and to coordinate the treatment process in a distributed way among several healthcare workers. We have implemented a system that supports consistency for distributed healthcare and integrates the treatment workflows into a healthcare workflow system. We use a clinical health care example to illustrate the workflow.
\end{abstract}

\section{CCS CONCEPTS}

- Information systems $\rightarrow$ Web applications; • Networks; - Computing methodologies $\rightarrow$ Modeling and simulation; - Computer systems organization $\rightarrow$ Distributed architectures;

\section{KEYWORDS}

Workflow management, High-level Petri nets, Pathways, Mobile Service Integration, Distributed Systems

\section{ACM Reference Format:}

Svetlana Boudko and Wolfgang Leister. 2017. Treatment Pathways as Petri Nets in Patient Workflow Management. In iiWAS '17: The 19th International Conference on Information Integration and Web-based Applications 85 Services, December 4-6, 2017, Salzburg, Austria. ACM, New York, NY, USA, 8 pages. https: //doi.org/10.1145/3151759.3151778

\section{INTRODUCTION}

Increased life expectancy and an ageing population in Europe challenge resource limitations in health care and prompt for advanced healthcare solutions. Digital technology and

Permission to make digital or hard copies of part or all of this work for personal or classroom use is granted without fee provided that copies are not made or distributed for profit or commercial advantage and that copies bear this notice and the full citation on the first page Copyrights for third-party components of this work must be honored

For all other uses, contact the owner/author(s).

iiWAS '17, December 4-6, 2017, Salzburg, Austria

(C) 2017 Copyright held by the owner/author(s).

ACM ISBN 978-1-4503-5299-4/17/12.

https://doi.org/10.1145/3151759.3151778 mobile health applications contribute to the sustainability and resilience of Europe's healthcare systems [13]. At the same time, mobile access to patient data by several healthcare stakeholders requires effective workflow management. In other words, the workflow process must be kept consistent with all stakeholders at all times. Workflow support is, therefore, crucial for coordination of patient care. In this paper, we present a solution for professional support of nurses in municipality health services, simplifying their task of obligatory recording of Electronic Health Records (EHR). For this, we use workflow management to model and develop treatment workflows, which are addressed in the paper as healthcare pathways. We have developed graphical workflow structures for describing tasks to be carried out, improving the quality and the uniformity of the health services provided and having access to the structured data. The specification for this system was developed in collaboration with the healthcare stakeholders.

The work proposed in this paper includes the following contributions: 1) We have designed patient treatment processes as workflows and implemented these workflows as Petri nets, which can be used as plug-ins. Thus, they can be easily added to or removed from the system. 2) We have developed a Petri net-based infrastructure that integrates these treatment workflows into a healthcare workflow system. It also supports consistency for a distributed healthcare system. Users of this healthcare system can remotely access the data of the Petri net using the Internet.

The implementated workflow management module combines the healthcare worker's workflow and the patient's healthcare pathways. Formalising pathways and workflow in one concept has not been done in this form before, and will be a breakthrough for workflow support in health care. In addition, the giving health personnel the access to information relevant to the current patient, and also to possible side-effects of medications, and inter-dependencies between diseases will improve healthcare systems.

The remainder of the paper is organised as follows: In Section 2, we introduce the theoretical background for our research and study related work. The design of the pathway model is presented in Section 3. A representative test scenario is outlined in Section 4, before discussing future work and concluding in Section 5. 


\section{BACKGROUND AND RELATED WORK}

The research field concerning workflow management that combines both the healthcare worker's workflow and the patient's healthcare pathways is not well explored. Thus, we review briefly healthcare pathways for patients that use care services, as well as workflow technologies that enable integration of pathways and workflows.

\subsection{Healthcare Pathways}

Healthcare pathways $[6,44,45]$ are multidisciplinary tools that can be applied for patients with predictable health development. Various tasks for health personnel are defined, optimised, and organised into time sequences. These are applied on a daily basis to patients that are in care or per visit in home care. A healthcare pathway can be seen as a workflow for treatment and care of a patient with a known diagnosis, such as one or multiple chronic diseases. The European Pathway Association [9] presents a definition of a care pathway including a literature list.

We define a treatment pathway as a structured multidisciplinary outline of anticipated care, placed in an appropriate time frame. It helps a patient with a specific profile and set of conditions or symptoms move progressively through a care experience towards a positive outcome [19].

The UK National Institute for Health and Care Excellence (NICE) [29] defines pathways for a variety of diseases as visual representations of recommendations where information on a topic is graphically linked to other topics.

The VIPS model [8] structures the essential elements in nursing as keywords to support the underlying nursing process. The VIPS model presents the nursing process model with $a$ ) assessment (nurse history, nurse status), b) nurse diagnosis, $c$ ) nursing goal, $d$ ) nursing intervention, and $e$ ) nursing outcome [11].

Ozcan et al. [31] and Tànfani and Testi [39] describe models and simulation methods for treatment. Healthcare pathways are also used for diseases that the elderly often suffer from $[34,37]$, for patients that are dismissed after having been hospitalised [20], or for patients with chronic diseases [7].

\section{$2.2 \quad$ Workflows}

A business process is a set of tasks that, when completed, achieves a business objective or goal. A workflow is an automation of such a process. An activity is a chunk of work within a process. In our work, we consider activities to be atomic, that is, they cannot be partially executed; thus, they need to be in a state that is either successfully completed or rolled back. Before an activity can be executed there might be certain preconditions that have to be met. After the successful execution of an activity, some postconditons apply.

The activities in a process can be required to be executed sequentially, or they can be executed simultaneously and asynchronously [40]. An activity can be mandatory or optional, and it can be restricted to be executed once or multiple times. Some activities can be executed anytime during a workflow,

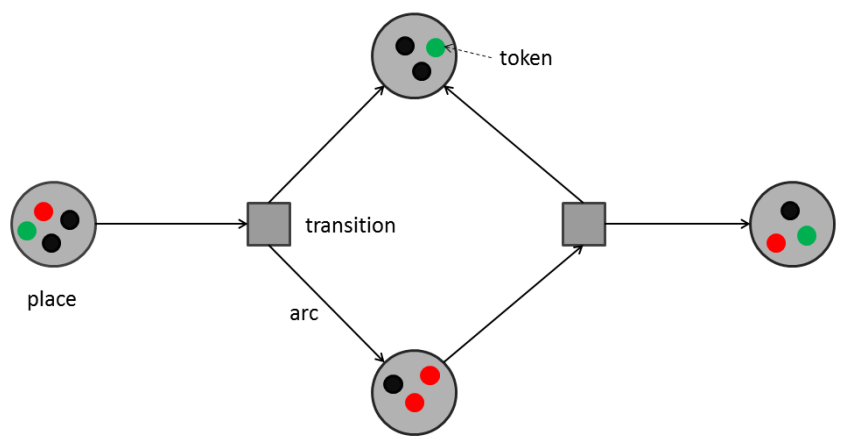

Figure 1: Example of a high-level Petri net.

while others depend on other activities. Further, activities within a workflow can be chained in various combinations, such as in sequence, in parallel, optionally, or repeatable.

A workflow can be modelled by various technologies, e.g., Event-driven Process Chains (EPCs) [41], Business Process Modeling Notation (BPMN) [30, 42], Unified Modeling Languages (UML), Bayesian networks [38], or Markov chains [27]. It has been shown that High-Level Petri Nets (HLPNs) have several key advantages over competing models [1]. We discuss this technology in the next subsection.

\subsection{High-Level Petri Nets}

Petri nets [32] were designed to model concurrency and synchronisation in distributed systems. Initially, Petri nets were used to model rather simple systems that are timeless and with resources of an identical type. Certainly, these limitations are incompatible with many real systems. A concise introduction to Petri nets and their application to workflow management is given by van der Aalst [40]. Petri nets are widely used for validation and verification in various application domains [17].

High-Level Petri Nets (HLPNs) were introduced by Jensen and Rozenberg [18] to overcome the mentioned limitations of the original Petri nets. HLPNs are mathematical models [16] that extend the original Petri net formalism [32] with the concepts of data types, time, and hierarchy. HLPN result in simple, precise, and scalable process models that are wellsuited for formal descriptions of all types of discrete systems. HLPNs can be applied to real processes as shown by, e.g., Kristoffersen and Boudko [22] and Kristoffersen et al. [23].

Similar to Petri nets, HLPNs are composed of four different types of elements: 1) places, 2) tokens, 3) transitions, and 4) arcs. Further, transitions can also represent sub-nets, thus hiding more complex functionality in one building block. Differently from Petri nets, tokens in HLPNs can carry complex information about the objects they represent.

Figure 1 shows a simple example of a HLPN. Detailed state information is stored in tokens, which are similar to objects or structures found in many programming languages. Tokens are a means of adding data to Petri nets. The static structure of a Petri net is a graph consisting of places and transitions connected by arcs. A transition controls the movement of 
tokens in a Petri net and can change the token data. Each transition has a guard expression, which is a Boolean function. If the output of the guard expression is evaluated to true the transition is fired.

An arc connects one place to one transition. It can be either an input arc, which is directed from a place to a transition, or an output arc, which is directed from a transition to a place. Every arc has an arc expression. In the case of an input arc, the arc expression describes the set of tokens necessary to enable its connected transition. These tokens will be removed from its connected place after the transition has occurred. In the case of an output arc, the arc expression describes a set of tokens deposited in its connected place when the transition occurs.

The entire dynamic structure of a Petri net consists of tokens stored in places. Thus, the global state of a Petri net at any particular point in time is represented by the tokens and the places that they are located in, collectively called the marking of the net. Transitions represent potential changes in state. Arcs and arc expressions determine precisely under which conditions these changes may occur and how they occur.

Previous work in the area of Petri net applications was mainly done for modelling in the industrial and communication domains with a focus on verification of hardware and software, analysing behavioural features of interactions in multi-agent systems, model checking of coordination protocols, as well as facilitating and monitoring of technological processes [5, 14, 21, 28, 35, 36].

\subsection{Workflow Support in Healthcare}

Few efforts have been invested in the development of workflow support for the healthcare domain. Mans [26] presented the use of workflow and its challenges in the health care domain. Hughes et al. [15] use Petri nets for planning and resource management in healthcare systems. Patient flows are modelled to provide healthcare managers with relevant information about requirements of new healthcare facilities and performance evaluation of existing facilities.

Bertolini et al. [4] proposed Petri nets to model collaboration of healthcare workflows and to verify functional and non-functional requirements of healthcare systems.

Augusto and Xie [2] proposed a modelling methodology for short-term planning and scheduling of health care activities. This methodology can also be used for simulation of health care systems.

To meet the concerns of patients and elderly people to live autonomously, Fanti et al. [10] presented a Petri net model to monitor their daily routines, detect the possible troubles and accidents, communicate with family, doctors and emergency services.

Whittaker et al. [43] proposed choice-point nets, which are an augmented form of Petri nets to analyse health-care protocols and to model probabilistic choices. The authors focused on how the choices among medical decisions can be simulated and determined.
Mahulea et al. [24, 25] showed that medical protocols can be modelled by state machine Petri nets. The authors do not use open Petri nets for their system. Thus, the resources remain unchanged and there is no real time interaction with the real world.

In our analysis, we recognise that the presented previous work does not address several important issues such as integrating healthcare workers' workflows and pathways and maintaining a set of pathways for patients with co-morbidities. These considerations motivate us to address these problems.

\section{DESIGNING PATHWAYS}

Treatment pathways, as defined in Section 2, are designed by medical specialists who think in terms of an overall plan. Such pathways are represented as a flowchart of states that the client or patient can be in. Flowchart can be divided into stages, and it can contain branches representing various alternative treatment paths. Treatment alternatives may succeed or fail, thereby determining the further path to be taken.

\subsection{Pathway Building Blocks}

Pathways are constructed from a list of interventions belonging to one of the following classes: $a$ ) assessments (questionnaires); b) measurements (taking data readings); c) tasks (e.g., feeding the patient); $d$ ) medication (administering or tracking); e) carer observations (free entry); $f$ ) randomly triggered manual alerts (e.g., fall detection); $g$ ) consent; $h$ ) sensorbased monitoring and alerts (e.g., lack of movement, too low room temperature).

Interventions have the following attributes: a) name; b) role (stakeholder responsible for the intervention, e.g., junior/senior nurse, client for self-administered outcome measurement); $c$ ) scheduling frequency; $d$ ) scheduling preference (e.g., time slot, day of the week, after meals); $e$ ) duration (the expected time allowed for each intervention); $f$ ) value (each intervention is given a single data value (logical or numeric) that will be compared to a status change parameter whenever it changes and an alert status assigned accordingly); g) alert status (each intervention is given a status, e.g., green (no action), yellow (admin), amber (low level, cumulative), red (immediate action on change), gold (achievement milestone)).

Unless specifically requested for an exception case, the default alert status for all interventions is green. At any point in time the status of an intervention is determined by the data values, attributes, and rules set by those who have paced the intervention into the library. For example, the status of a weight measure is determined by the alert thresholds in that measure.

The workflow process translates the assigned interventions into events. This means that, depending on its frequency, each intervention produces one or more events. These events need to be scheduled and authorised. The completion of the event has to be confirmed and registered by the system and can result in assigning new pathways to the patient. 


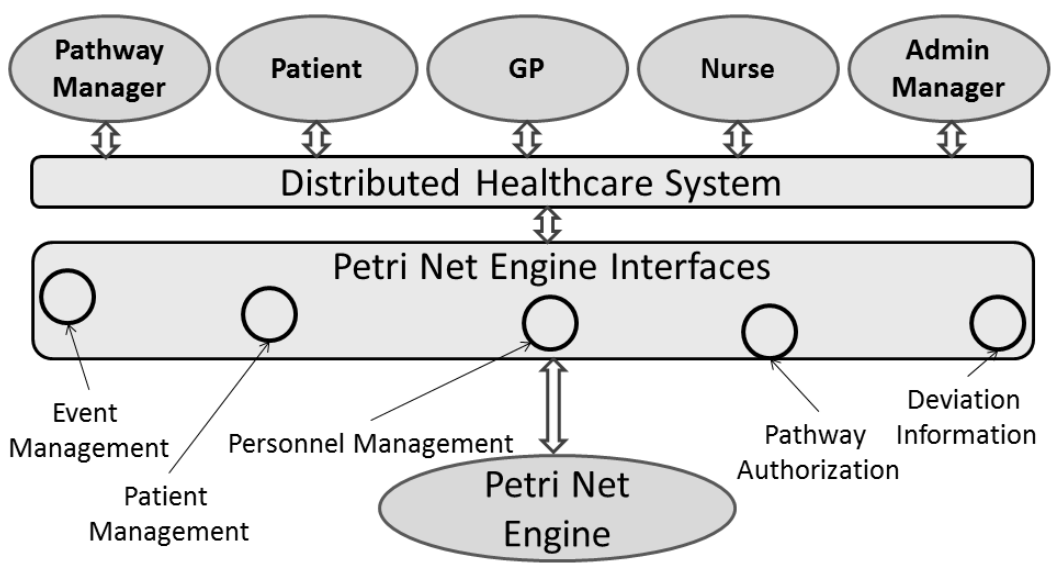

Figure 2: System Architecture of the Distributed Healthcare System. The external components communicate with the Petri Net using the Interface Layer.

\subsection{System Architecture}

The workflow support module for pathways is part of a larger system that a supports healthcare personnel in their daily work. This system implements functionality for the user interface, data storage, data communication, etc. The system, depicted as Distributed Healthcare System in Figure 2, interacts with the workflow management module, implemented by the Petri net Engine.

The real world is composed of several external components that represent patients and healthcare stakeholders. These components can communicate with the Petri net via the Distributed Healthcare System, either using wired or mobile connection. These components are the following: 1) the Pathway Manager component, 2) the Admin Manager component, 3) the Patient component, 4) the Nurse component, and 5) the GP component. The Distributed Healthcare System can be used stand-alone, but then without the support of the guidance and consistency functionality provided by the Petri net engine. When the Petri net functionality is activated, the Petri net engine manages interactions, which avoids potential consistency problems.

The Petri net engine stores and executes Petri nets containing meta-data. In this work, we use an idea of an open Petri net presented by Baldan et al. [3]. Such nets contain a set of places that have interfaces to the external environment. The environment can add or remove tokens from those places using these interfaces, which provide a means of communication between the Petri net model and the real world.

We use the Petri net engine Andromeda [22] that has been applied earlier in several application areas, among others to aviation and the printing industry. The Petri net engine allows meta-data to be stored in hierarchical coloured Petri nets. Further, it includes a generic protocol that enables a client to insert tokens into the net and query the net about tokens and their contained meta-data. The system communicates with the Petri net via a set of interfaces, each consisting of one or more Petri net places.

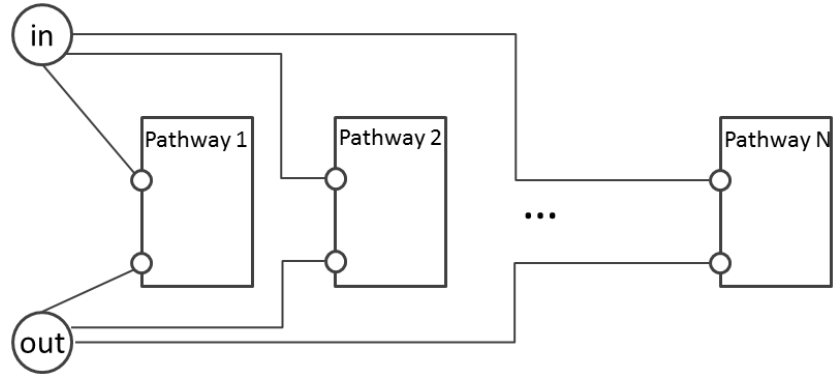

Figure 3: Pathway manager subnet. New pathways can be easily added to the subnet while existing pathways can be removed or altered.

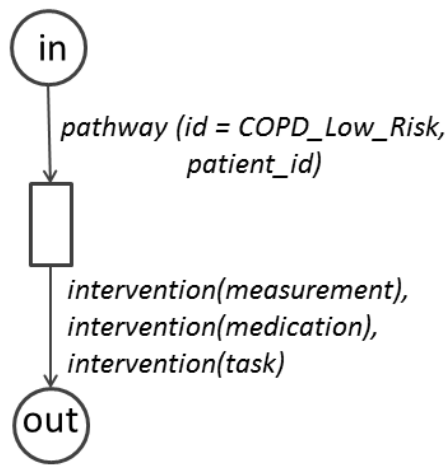

Figure 4: Example of a pathway subnet with the COPD Low Risk pathway. The input is a pathway token with the pathway id and the patient's id. The output is a set of tokens representing interventions that are part of a treatment plan for COPD low risk patients. 


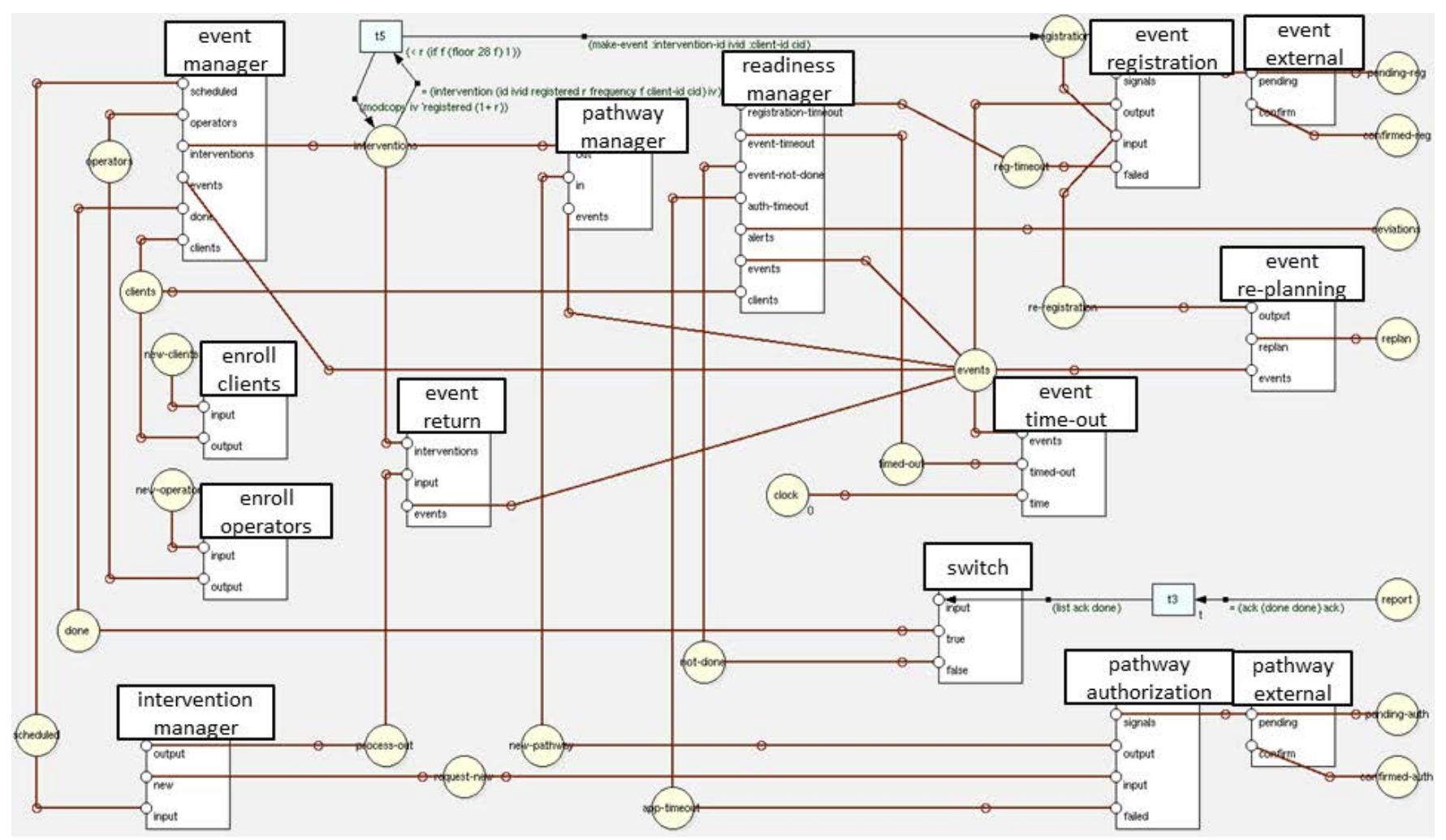

Figure 5: Petri net design.

\subsection{Petri Net Design}

The Petri net implements the workflow for healthcare stakeholders. Its design is depicted in Figure 5. Treatment pathways are designed as separate modules that can be added to or removed from the system. A patient can be assigned to several pathways, which represent the patient's treatment plan. If the treatment process for the patient is changed, new pathways can be added to the patient's treatment plan. Pathways that are no longer valid can be deleted.

Tokens. Tokens and their respective places define the status of the current workflow. Besides several auxiliary tokens, our implementation defines five main token classes for patients, personnel, pathway, intervention, and (external) events. As we think of more generic applications for the designed Petri net, tokens representing patients are further referred as client tokens. Tokens representing healthcare stakeholders are referred as operator tokens. A pathway token contains the fields for client id, alert level, and the approval status of this pathway. An intervention token implements the fields for the pathway id of the related pathway, frequency, threshold, and further intervention specific values. Events are represented by an event token. The token specifies the intervention that triggers this event, the patient who is going to receive the care, and the personnel who performs it. It specifies whether the event has been scheduled or not, and if so, when the event has to happen. It also has an alert status. For communication with external processes, we have defined two types of tokens: signals that convey information from the Petri net to the external processes, and acknowledgements that convey information in the opposite direction. Note that all token classes contain a field for a unique id.

The Petri net implements the following functionality:

Pathway Management. The Petri net functions as a plugboard for treatment pathways that are implemented as Petri net modules. The plugboard is developed as a separate subnet named pathway manager, shown in Figure 3. An example of a treatment pathway module is depicted in Figure 4.

In case the net decides that new pathways should be assigned to a patient, new pathway tokens for this patient are created. Pathways that are added to the patient's treatment plan by the net require explicit authorisation by Pathway Managers or GPs. After the pathway is authorised, the transition fires, and the pathway-token is inserted into the in-place of the pathway manager subnet. This results in the creation of intervention tokens for the pathway and the patient. The out place of the pathway manager subnet is connected with the interventions place of the main Petri net by a place fusion. The corresponding event tokens are also created.

Patient Management. The Petri net keeps track of patients. It is possible to enrol new patients by depositing requests 
representing their IDs in the new-clients place. The information is processed by the enroll-clients subnet, where a client token is created and deposed in the clients place. Patients can be monitored by inspecting the clients place including the alarm status for this patient. The clients place can be used to obtain a list of the existing patients, or to remove a patient from the system.

Personnel Management. Personnel can be added to, monitored or removed from the system. Tokens that represent new staff are inserted in new-operators, processed by the enroll operators subnet, and are kept in the operators place.

Event Management. This module implements registration, re-planning, and reporting of events. For each patient and intervention, the net produces one or more event tokens. Each token represents an action that might occur in the real world. Some classes of events, such as measurements and medications, are scheduled to be performed at a particular time by a particular operator. Other classes of events, such as manual alerts and sensor-based alerts, are set up in advance, but it is uncertain if and when they will occur. The purpose of the event registration is to synchronise both kinds of events between the Petri net and the system. After an event token is created, it is sent to the event registration subnet for further scheduling. For each event that requires registration, a signal token is deposited into the pending-reg place, where it can be inspected by the personnel. The event re-planning allows the system to make changes to events that are already registered. The purpose of the event reporting is to inform the net of scheduled events that have been completed, successfully or not, and unscheduled events that have occurred. The system reports the completion or occurrence of an event by depositing an acknowledgement token in the report place.

Deviation Information. The purpose of the deviation information is to present information on deviations in condensed form. The system can retrieve this information and present it to the Admin Manager component. Four types of deviations are accounted for: $a$ ) an event pending registration was ignored and timed out; $b$ ) a scheduled event was not reported, or it passed its scheduled time plus a pre-defined slack time; $c$ ) a scheduled event was not successfully completed; and $d$ ) a pathway pending authorisation was ignored and timed out.

\section{SCENARIO FOR THE USE OF PATHWAYS}

Using a scenario considering patients with Chronic Obstructive Pulmonary Disease (COPD) [12], we show how the work flow engine is used and the HLPNs are modelled. For this scenario, we defined the following pathways:

COPD Measure Pathway. This pathway measures the disease activity. It consists of a measurement intervention that is performed by a nurse. Consent from the patient is required. The intervention is performed once. Depending on the result, the patient can be assigned to the COPD Low Risk Pathway or COPD High Risk Pathway, which are both described below.

COPD Low Risk Pathway. This pathway for low risk patients consists of the following three interventions:

- Intervention $A$ is the set-up for the daily activities to be performed by a nurse. It is performed once.

- Intervention $B$ is a medication prescription performed by a GP. Consent from the patient is required, and the intervention is performed once.

- Intervention $C$ is a COPD measurement that is performed monthly. Depending on the result, the patient can continue the COPD High Risk Pathway or can be assigned to the COPD Low Risk Pathway.

COPD High Risk Pathway. This pathway for high risk patients consists of the following four interventions:

- Intervention $D$ is the set-up for the daily activities to be performed by a nurse. It is performed once.

- Intervention $E$ is a medication prescription performed by a GP. Consent from the patient is required, and the intervention is performed once.

- Intervention $F$ is a COPD measurement that is performed weekly. Depending on the result, the patient can continue the COPD High Risk Pathway or can be assigned to the COPD Low Risk Pathway.

- Intervention $G$ is a COPD assessment. It consists of a questionnaire performed monthly by a nurse.

\section{Scenario Description}

We assume that the patient is already admitted into the health care system, and the preliminary evaluation for the diagnosis is performed. The patient is assigned to the COPD Measure Pathway. The COPD measurement indicates that the patient fits to the COPD Low risk pathway. A token for the COPD Low Risk Pathway is created. The GP receives a notification to approve the COPD Low risk pathway for this patient. After the pathway is approved, tokens for the interventions of this pathway are created and corresponding events are scheduled. Further, the medication is prescribed. Then, the nurse visits the patient and sets up the daily activities. After these two events are completed, the routine COPD measurement is scheduled monthly with subsequent events.

The nurse who is scheduled to perform the routine COPD measurement is not available to attend the event and informs the system about this fact. A signal token that indicates that an event needs re-planing is inserted. The administrator is notified that a calendar precondition no longer is met. Thus, the administrator selects alternative available staff with a valid role at a suitable time slot. An event with new time slot and new personnel is deployed.

The nurse arrives to perform the routine COPD measurement. The measurement is done and registered. The measured value is below the predefined threshold. This indicates that 
the patient needs to be assigned to the COPD High Risk Pathway. As a consequence, a new pathway token is created.

Further, an alert notification is sent to the GP. The new pathway is approved with its intervention tokens created, and corresponding events are deployed. The COPD Low Risk Pathway is no longer valid for this patient and the related tokens for this pathway and its interventions are deleted.

\section{CONCLUSION}

We have presented how HLPN can be used to integrate the patients' treatment pathways and healthcare personnel workflows into one common workflow. The information to support the various healthcare stakeholders can be retrieved from the Petri net through interfaces that connect the Petri net to the real world. The design of the infrastructure have been validated through several healthcare scenarios, where we presented one of them.

At present, we have implemented treatment pathways for patients with COPD. Other treatment pathways can be easily added to the infrastructure after they are modeled as Petri nets. Prototype tools are now available to model and add new pathways.

As future work, besides adding more pathways to a library and improving the tools to design the pathways, the management of Petri net metadata needs further development. Scalability of the proposed solution is a further, important issue that should be addressed in our future work. With many requests going through the system, the Petri net engine can easily become a bottleneck. Therefore, implementing the engine as, i.e., a cluster of servers can be considered. Another interesting research topic could be to decouple the services based on the implemented subnets and to implement these as separate Petri nets that communicate with each other through external processes. Finally, the integration between the Petri net and optimisation components that support planning and scheduling of healthcare workers, is necessary for the practical use of our implementation.

\section{ACKNOWLEDGMENT}

The work presented in this paper has been conducted as a part of the Eurostars Project E!9080 EHRPathways. The authors thank Thor Kristoffersen for implementing the Andromeda system and major parts of the Petri net models. The authors thank Heidi Camilla Mork for her initial work on the Petri net models for the health pathways. The design and implementation of the basis system was performed by the UK-based company Alloy Ltd. and the Norwegian companies NORNIR AS and Hospital Organiser AS for WellTogether Ltd. The authors wish to thank Thorhallur Gudmundsson, Gus Desbarats, Pål Kristian Levang, Halvor Kalve, and the team of developers for their support.

\section{REFERENCES}

[1] A. Alhroob, K. Dahal, and A. Hossain. 2010. Transforming UML sequence diagram to High Level Petri Net. In 2nd Intl Conf Software Technology and Engineering (ICSTE), Vol. 1. IEEE, V1-260 - V1-264. https://doi.org/10.1109/ICSTE.2010.5608842
[2] V. Augusto and X. Xie. 2014. A Modeling and Simulation Framework for Health Care Systems. IEEE Transactions on Systems, Man, and Cybernetics: Systems 44, 1 (Jan 2014), 30-46. https://doi.org/10.1109/TSMC.2013.2239640

[3] Paolo Baldan, Andrea Corradini, Hartmut Ehrig, and Barbara König. 2008. Open Petri Nets: Non-deterministic Processes and Compositionality. In Graph Transformations: 4th International Conference, ICGT 2008, Leicester, United Kingdom, September 7-13, 2008. Proceedings, Hartmut Ehrig, Reiko Heckel, Grzegorz Rozenberg, and Gabriele Taentzer (Eds.). Springer Berlin Heidelberg, Berlin, Heidelberg, 257-273. https://doi.org/10.1007/ 978-3-540-87405-8_18

[4] Cristiano Bertolini, Zhiming Liu, and Jiří Srba. 2013. Verification of Timed Healthcare Workflows Using Component Timed-Arc Petri Nets. In Foundations of Health Information Engineering and Systems: Second International Symposium, FHIES 2012, Paris, France, August 27-28, 2012. Revised Selected Papers, Jens Weber and Isabelle Perseil (Eds.). Springer, Berlin, Heidelberg, 19-36. https://doi.org/10.1007/978-3-642-39088-3_2

[5] J. R. Celaya, A. A. Desrochers, and R. J. Graves. 2007. Modeling and analysis of multi-agent systems using petri nets. In 2007 IEEE International Conference on Systems, Man and Cybernetics. 1439-1444. https://doi.org/10.1109/ICSMC.2007.4413960

[6] Leentje de Bleser, Roeland Depreitere, Katrijn de Waele, Kris Vanhaecht, Joan Vlayen, and Walter Sermeus. 2006. Defining pathways. J Nursing Management 14, 7 (2006), 553-563. https: //doi.org/10.1111/j.1365-2934.2006.00702.x

[7] Svin Deneckere, Martin Euwema, Cathy Lodewijckx, Massimiliano Panella, Walter Sermeus, and Kris Vanhaecht. 2012. The European quality of care pathways (EQCP) study on the impact of care pathways on interprofessional teamwork in an acute hospital setting: study protocol: for a cluster randomised controlled trial and evaluation of implementation processes. Implement Sci 7, 1 (2012), 47

[8] Margareta Ehnfors, Ingrid Thorell-Ekstrand, and Anna Ehrenberg. 1991. Towards basic nursing information in patient records. Vard i Norden 21, 3-4 (1991), 12-31.

[9] European Pathway Association. 2017. Care Pathways. interactive web site. (2017). http://e-p-a.org/care-pathways accessed: 7 August, 2017.

[10] M. P. Fanti, A. M. Mangini, W. Ukovic, J. J. Lesage, and K. Viard. 2014. A Petri net model of an integrated system for the Health Care At Home management. In 2014 IEEE International Conference on Automation Science and Engineering (CASE). 582-587. https://doi.org/10.1109/CoASE.2014.6899385

[11] Jan Florin, Anna Ehrenberg, Margareta Ehnfors, and Catrin Bjørvell. 2013. A comparison between the VIPS model and the ICF for expressing nursing content in the health care record. $I$. J. Medical Informatics 82, 2 (2013), 108-117.

[12] Kevin Gruffydd-Jones. 2012. GOLD guidelines 2011: what are the implications for primary care? Primary Care Respiratory Journal 21, 4 (December 2012), 437-441. https://doi.org/10.4104/ pcrj.2012.00058

[13] Health and Food Safety Directorate General. 2017. Public Health: Midterm Review of Digital Single Market Strategy includes areas for enhanced action in digital health and care. e-News. (10 May 2017). http://ec.europa.eu/newsroom/sante/ newsletter-specific-archive-issue.cfm?newsletter_service_id $=$ $327 \&$ newsletter_issue_id $=3546 \&$ page $=1 \&$ fullDate $=$ Thu $\% 2005 \%$ 20 Oct $\% 202017$

[14] Steve P. Hostettler, Alexis A. Marechal Marin, Alban Linard, Matteo Risoldi, and Didier Buchs. 2011. High-Level Petri Net Model Checking with AlPiNA. Fundamenta Informaticae 113, 3-4 (2011), 229-264. ID: unige:18361.

[15] M. Hughes, E. R. Carson, M. Makhlouf, C. J. Morgan, and R. Summers. 1998. A Petri net based model of patient-flows in a progressive patient-care system. In Proceedings of the 20th Annual International Conference of the IEEE Engineering in Medicine and Biology Society. Vol.20 Biomedical Engineering Towards the Year 2000 and Beyond (Cat. No.98CH36286), Vol. 6. 3048-3051 vol.6. https://doi.org/10.1109/IEMBS.1998.746134

[16] Kurt Jensen. 1997. Coloured Petri Nets - Basic Concepts, Analysis Methods and Practical Use. EATCS, Monographs on Theoretical Computer Science, Vol. 1. Springer-Verlag. Basic Concepts.

[17] Kurt Jensen and Lars M. Kristensen. 2009. Coloured Petri Nets: Modelling and Validation of Concurrent Systems (1st ed.). Springer Publishing. 
[18] Kurt Jensen and Grzegorz Rozenberg (Eds.). 1991. High-level Petri Nets: Theory and Application. Springer-Verlag, London, $\mathrm{UK}, \mathrm{UK}$.

[19] Robert Jones and Fiona Jenkins. 2011. Key Tools and Techniques in Management and Leadership of the Allied Health Professions (1 edition ed.). CRC Press, London.

[20] K. Katsaliaki, S. Brailsford, D. Browning, and P. Knight. 2005 Mapping care pathways for the elderly. J Health Organ Manag. 19, 1 (2005), 57-72.

[21] Gitosree Khan, Sabnam Sengupta, and Anirban Sarkar. 2017 Formal Modeling of Enterprise Cloud Bus System: A High Level Petri-Net Based Approach. In Requirements Engineering for Service and Cloud Computing, Muthu Ramachandran and Zaigham Mahmood (Eds.). Springer International Publishing, Cham, 121149. https://doi.org/10.1007/978-3-319-51310-2_6

[22] Thor Kristoffersen and Svetlana Boudko. 2006. Simplified Workflow Management with Metadata-Enhanced Petri Nets. In Proc IEEE Intl Conf Systems, Man, and Cybernetics, Vol. 4. 27292735. https://doi.org/10.1109/ICSMC.2006.385286

[23] Thor Kristoffersen, Anders Moen, and Hallstein A. Hansen. 2003 Extracting High-Level Information from Petri Nets: A Railroad Case. Proceedings of the Estonian Academy of Physics and Mathematics 52, 4 (December 2003), 378-393.

[24] Cristian Mahulea, Liliana Mahulea, José Manuel GarcÃa-Soriano, and José-Manuel Colom. 2014. Petri nets with resources for modeling primary healthcare systems. In 2014 18th International Conference on System Theory, Control and Computing (IC$S T C C)$. 639-644. https://doi.org/10.1109/ICSTCC.2014.6982489

[25] Cristian Mahulea, Liliana Mahulea, Juan Manuel García Soriano, and José Manuel Colom. 2017. Modular Petri net modeling of healthcare systems. Flexible Services and Manufacturing Journal (11 Mar 2017), 1-29. https://doi.org/10.1007/s10696-017-9283-9

[26] Ronny S. Mans. 2011. Workflow Support for the Healthcare Domain. Ph.D. Dissertation. Technische Universiteit Eindhoven.

27] Andrey Markov. 1971 (1906). Extension of the Limit Theorems of Probability Theory to a Sum of Variables Connected in a Chain. In Dynamic Probabilistic Systems (Volume I: Markov Models), R. Howard (Ed.). John Wiley \& Sons, Inc., New York City, Chapter Appendix B, 552-577.

[28] J. M. Mendes, P. Leitao, A. W. Colombo, and F. Restivo. 2008. High-Level Petri Nets control modules for service-oriented devices: A case study. In 2008 34th Annual Conference of IEEE Industrial Electronics. 1487-1492. https://doi.org/10.1109/IECON.2008. 4758173

[29] National Institute for Health and Care Excellence (NICE). 2017 NICE pathways. interactive web site. (2017). https://pathways. nice.org.uk/ accessed: 7 August, 2017.

[30] OMG. 2011. Documents associated with Business Process Model and Notation (BPMN), Version 2.0. Technical Report. Object Management Group (OMG)/Business Process Management Initiative (BPMI). http://www.omg.org/spec/BPMN/2.0/ accessed: 7 August, 2017

[31] Yasar A. Ozcan, Elena Tànfani, and Angela Testi. 2011. A simulation-based modeling framework to deal with clinical pathways. In Proceedings of the Winter Simulation Conference (WSC '11). Winter Simulation Conference, Phoenix, Arizona, 1190-1201.

[32] Carl Adam Petri. 1962. Fundamentals of a Theory of Asynchronous Information Flow.. In IFIP Congress. 386-390.

[33] Carl Adam Petri. 1962. Kommunikation mit Automaten Schriften des IIM Nr. 2. Bonn: Institut für Instrumentelle Mathematik. in German.

[34] I Philp, J. Houghton, M. Roland, V. Leah, and R. Stephens 2012. Frail Elderly Pathway. Rapid Response Review. Cambridge University Hospitals Foundation Trust.

[35] J. Pinto, J. M. Mendes, P. Leitdo, A. W. Colombo, A. Bepperling, and F. Restivo. 2009. Decision support system for Petri nets enabled automation components. In 2009 7th IEEE International Conference on Industrial Informatics. 289-294. https://doi.org/ 10.1109/INDIN.2009.5195819

[36] Albert Pla, Pablo Gay, Joaquim Meléndez, and Beatriz López. 2014. Petri net-based process monitoring: a workflow management system for process modelling and monitoring. Journal of Intelligent Manufacturing 25, 3 (01 Jun 2014), 539-554. https://doi.org/10.1007/s10845-012-0704-z

37] Delphine Rossille, Marc Cuggia, Aude Arnault, Jacques Bouget, and Pierre Beux. 2008. Managing an emergency department by analysing HIS medical data:a focus on elderly patient clinical pathways. Health Care Management Science 11, 2 (2008), 139146. https://doi.org/10.1007/s10729-008-9059-6

[38] Stuart Russel and Peter Norvig. 2003. Artificial Intelligence - a modern approach. Pearson Education.

[39] Elena Tànfani and Angela Testi. 2012. A simulation-based decision support tool to analyze clinical pathways in hospital. In Advanced Decision Making Methods Applied to Health Care, Elena Tànfani and Angela Testi (Eds.). International Series in Operations Research \& Management Science, Vol. 173. Springer Milan, 191-211. https://doi.org/10.1007/978-88-470-2321-5_12

[40] Wil M. P. van der Aalst. 1998. The Application of Petri Nets to Workflow Management. The Journal of Circuits, Systems and Computers 8, 1 (1998), 21-66.

[41] Willibrordus Martinus Pancratius van der Aalst. 1999. Formalization and Verification of Event-driven Process Chains. Information E Software Technology 41, 10 (1999), 639-650.

[42] Matthias Weidlich, Gero Decker, Alexander Großkopf, and Mathias Weske. 2008. BPEL to BPMN: The Myth of a Straight-Forward Mapping. In OTM Conferences (1) (Lecture Notes in Computer Science), Robert Meersman and Zahir Tari (Eds.), Vol. 5331. Springer, 265-282.

[43] S. J. Whittaker, K. Rudie, and J. McLellan. 2015. An Augmented Petri Net Model for Health-Care Protocols. IEEE Trans. Automat. Control 60, 9 (Sept 2015), 2362-2377. https: //doi.org/10.1109/TAC.2015.2409932

[44] Karen Zander. 2002. Integrated care pathways: eleven international trends. J Integrated Care Pathways 6 (2002), 101-107.

[45] Karen Zander and Kathleen Bower. 1987. Nursing Case Management, Blueprint for Transformation. New England Medical Center, Boston. 